\title{
Plasma Kynurenine to Tryptophan Ratio Is Negatively Associated with Linear Growth of Children Living in a Slum of Bangladesh: Results from a Community-Based Intervention Study
}

\author{
Md. Amran Gazi, ${ }^{1 \star}$ Subhasish Das, ${ }^{1}$ Md. Abdullah Siddique, ${ }^{2}$ Md. Ashraful Alam, ${ }^{1}$ Shah Mohammad Fahim, ${ }^{1}$ Md. Mehedi Hasan, ${ }^{1}$ \\ Farzana Hossaini, ${ }^{1}$ Md. Mamun Kabir, ${ }^{2}$ Zannatun Noor, ${ }^{2}$ Rashidul Haque, ${ }^{2}$ Mustafa Mahfuz, ${ }^{1}$ and Tahmeed Ahmed ${ }^{1,3}$ \\ ${ }^{1}$ Nutrition and Clinical Services Division, International Centre for Diarrheal Disease Research, Bangladesh (icddr,b), Dhaka, Bangladesh; ${ }^{2}$ Emerging \\ Infection and Parasitology Laboratory, International Centre for Diarrheal Disease Research, Bangladesh (icddr,b), Dhaka, Bangladesh; ${ }^{3}$ James P. \\ Grant School of Public Health, BRAC University, Dhaka, Bangladesh
}

\begin{abstract}
Chronic exposure to infectious agents results in environmental enteric dysfunction-a significant contributor to childhood stunting. Low plasma tryptophan (TRP), increased kynurenine (KYN), and KYN-TRP (KT) ratio are associated with infections and chronic immune activation. We postulated that both these conditions are interlinked, and therefore aimed to identify the association between KT ratio and the linear growth of Bangladeshi children. A total of 480 stunted and at risk of being stunted children aged 12-18 months were enrolled and provided nutrition intervention for 90 days. Plasma samples were assessed using liquid chromatography tandem mass spectrometry to measure TRP and KYN concentrations. Multivariable linear regression with generalized estimating equations was applied to analyze association between the KT ratio and linear growth. Tryptophan, KYN, and KT ratio were significantly higher in stunted children than in children at risk of being stunted both at baseline and at the end of nutrition intervention. Following intervention, the median (interquartile range [IQR]) KYN concentration was significantly reduced from $4.6(3.6,5.4) \mu \mathrm{mol} / \mathrm{L}$ to $3.9(0.3,7.6) \mu \mathrm{mol} / \mathrm{L}$, and median (IQR) KT ratio decreased from $104(80.9,131)$ to $92.8(6.6,247)$ in stunted children. We also found KT ratio to be negatively associated (coefficient $=-0.7 ; 95 \% \mathrm{Cl}=-1.13,-0.26 ; P$-value $=0.002$ ) with linear growth. In addition, $\mathrm{KYN}$ and KT ratio were positively correlated with fecal neopterin and plasma C-reactive protein, whereas TRP was negatively correlated with both of these biomarkers and alpha-1-acid glycoprotein. Our findings imply that KT ratio is associated in the pathophysiology of stunting as well as with biomarkers of inflammation in Bangladeshi children.
\end{abstract}

\section{INTRODUCTION}

Stunting is highly prevalent, especially in lower income countries, affecting an estimated 149 million children younger than 5 years, worldwide. ${ }^{1}$ It is an ailment where multiple pathological conditions manifested by linear growth faltering can increase morbidity, impaired brain development, as well as development of chronic diseases in adulthood. ${ }^{2}$ Short stature is common in low- and middle-income countries; thus, it often remains unrecognized and seems to be "normal" and taken for granted. ${ }^{2}$ It is difficult to identify visually and is not customarily evaluated by primary healthcare facilities. This exposes a noticeable number of children to the risk of being stunted. ${ }^{2}$ Living in poor hygiene and sanitary conditions may cause gut dysfunction, referred to as environmental enteric dysfunction (EED), a subclinical inflammatory condition of the small intestine. ${ }^{3}$ Growth retardation, gut microbiota alteration, and reduced vaccine responsiveness are thought to be the predominant consequences of EED. ${ }^{4}$ Exposures to environmental challenges impose metabolic stress that induces either nonspecific or more specific immune responses when infections occur. ${ }^{5}$ In the most severe infections, such exposures lead to the complete alteration of metabolic priorities, and to an underlying change in the requirements of protein, amino acids (AAs), and nitrogen. ${ }^{5}$

Tryptophan (TRP), a plant-derived essential AA (EAA), is needed to support growth and health in humans. ${ }^{6}$ It produces bioactive compounds via niacin and serotonin synthetic pathways, and serves as a precursor for protein synthesis. ${ }^{6}$ However, TRP is mostly catabolized by an enzyme called indoleamine $2,3-$

*Address correspondence to Md. Amran Gazi, Nutrition and Clinical Services Division, International Centre for Diarrhoeal Disease Research (icddr,b), 68 Shaheed Tajuddin Ahmed Ave., Mohakhali, Dhaka-1212, Bangladesh. E-mail: amran.gazi@icddrb.org dioxygenase (IDO), induced by interferons, leading to the production of kynurenine (KYN). ${ }^{7}$ Reduction of TRP concentration and formation of the immunomodulatory metabolite KYN potentially impede T-cell proliferation and induce T-cell apoptosis. ${ }^{7}$ Immune activation thus gives rise to the formation of KYN and reduction of TRP, and a higher KYN-TRP (KT) ratio indicates a systemic immune response. ${ }^{8}$ The burden of infectious diseases is greater in children with stunting. ${ }^{9}$ Therefore, metabolic needs for these children require higher energy and AAs, which may divert EAAs to keep the immune system active at the expense of growth. ${ }^{10}$ For example, infestation with intestinal parasites increases the dietary requirements of lysine by $20 \%$ in undernourished children. ${ }^{11}$ Study also showed that chronic bacteria causing disease associated with wasting increased the protein turnover by nearly $40 \%$ when compared with uninfected subjects. ${ }^{12}$

Moreover, children in developing countries usually have limited access to animal source foods which are the richest sources of EAAs. ${ }^{13}$ Using a metabolomic approach, a study in rural Malawi showed that more than $80 \%$ children with stunting had reduced levels (15-20\%) of all nine EAAs, compared with children with normal growth. ${ }^{14}$ Dietary restriction of these EAAs and their effects have also been explored in animal studies. ${ }^{10}$ Restriction of dietary TRP resulted in severe growth retardation of young rats and pregnant rats, thus deprived of dietary TRP-delivered growth-retarded newborns. ${ }^{15}$ Consequently, it was seen that mice with a mutation in TRP transport pathway suffered from malnutrition and that supplementation of TRP could mitigate associated problems. ${ }^{16}$

The KT ratio has been used as a systemic inflammatory marker in conditions such as sepsis, type 2 diabetes, obesity, inflammatory bowel disease, and immunodeficiency syndrome. ${ }^{17-21}$ The KT ratio has also been found to be positively associated with the growth deficits in children in Peru and Tanzania. ${ }^{22}$ In addition, serotonin/TRP and KT ratios were 
found to be positively correlated with gut permeability in Malawian children. ${ }^{23}$ Tryptophan, after being transformed to KYN or serotonin, remains no longer available to synthesize protein; hence, their ratios to TRP can be used as a proxy indicator of inflammatory conditions. In children living in slum where infections occur synchronously, the cascade of immune response to these infections is presumed to have an impact on child development and growth. ${ }^{24}$ Therefore, we hypothesized that KT pathway-mediated inflammation that causes insufficiency in EAAs may be a limiting factor for linear growth in children. The present study aimed to assess the role of KYN pathway of TRP metabolism in linear growth of Bangladeshi children.

\section{MATERIALS AND METHODS}

Study site, participants, and ethical consideration. To perform this analysis, we have used data from the ongoing Bangladesh EED. This study is being conducted among slumdwelling residents in Mirpur, Dhaka, Bangladesh. The study protocol has already been published elsewhere. ${ }^{25}$ The primary objective of this study is to validate noninvasive biomarkers of EED and investigate the relationship of those biomarkers with stunting. Understanding the mechanism and pathophysiology of EED is also an important objective of the study. Children aged between 12 and 18 months were recruited for an intervention period of 3 months. The intervention included $150 \mathrm{~mL}$ of whole milk, an egg, micronutrient powder, and also nutritional counseling for 90 feeding days. The cohort consists of stunted (length-for-age $Z$-scores [LAZ] <-2) and at risk of stunting (LAZ $<-1$ to -2$)$ children. Data were taken from 480 children for this particular analysis. The Institutional Review Board of International Centre for Diarrhoeal Disease Research, Bangladesh approved the research protocol (protocol no PR-16007). Informed written consents were obtained from parents/caregivers of the participants before enrolment of their children to the study.

Data collection, specimen collection, and storage. Trained field staff measured the anthropometry following standard operating procedures. Weights and lengths of the participants were measured before and after the nutritional intervention along with mother's height using standard scales (Seca GmbH \& Co. KG., Hamburg, Germany). Measuring equipment was calibrated daily to maintain quality. In addition, uniformity of anthropometric measurement was also ensured by providing refresher training. Finally, intra-class correlation coefficient was estimated every 3 months. The WHO anthropometry calculator was used to calculate the indices of nutritional status, namely, weight-forage Z-score (WAZ), weight-for-height Z-score (WHZ), and LAZ, both at baseline and after nutrition intervention. Stool and blood samples were collected at enrolment and at the end of nutrition intervention. To separate the plasma, blood samples were centrifuged at 4,000 rotations per minute (rpm) for 10 minutes. Aliquots of stool and plasma were immediately frozen at $-80^{\circ} \mathrm{C}$, pending analysis.

Determination of TRP and KYN concentrations and KT ratio calculation. Reference compounds for TRP, KYN, and TRP-d5 were procured from Sigma (St. Louis, MO). Kynurenined4 was purchased from TLC pharmaceuticals (Newmarket, Ontario, Canada). Acetonitrile, methanol, tetrahydrofuran, isopropanol, formic acid, ammonium formate, and other reagents or solvents were high-performance liquid chromatography grade or analytical grade. The mobile phase A contained acetonitrile:tetrahydrofuran: $25 \mathrm{mM}$ ammonium formate:formic acid in a ratio of 9:75:16:0.3 (v), whereas the mobile phase B contained acetonitrile: $100 \mathrm{mM}$ ammonium formate in a ratio of 20:80. Standards for $\mathrm{L}-\mathrm{TRP}$ and $\mathrm{L}-\mathrm{KYN}$ were made at $1 \mathrm{mg} / \mathrm{mL}$ in $50 \%$ (vol/vol) acetonitrile in water. Serial dilutions were prepared in water to reach a seven-point calibration curve with ranges of TRP (10-1,000 ng/ $\mathrm{mL}$ ) and KYN (2-200 ng/mL). The internal standard was prepared with TRP-d5 and KYN-d4 at a concentration of $250 \mathrm{ng} / \mathrm{mL}$ and 50 $\mathrm{ng} / \mathrm{mL}$, respectively.

$20 \mu \mathrm{L}$ human plasma samples were de-proteinized by adding methanol/acetonitrile (1:1) solvent. Then, $100 \mu \mathrm{L}$ internal standard working solution was added, followed by vortexing for 10 minutes and centrifugation at 13,000 rpm for 10 minutes. The supernatant was separated and filtered with a $0.2-\mu \mathrm{m}$ nylon filter. Finally, $10 \mu \mathrm{L}$ samples were administered into the liquid chromatography with tandem mass spectrometry (LC-MS/MS) system. Flow rate for the system was set at $0.6 \mathrm{~mL} /$ minute, and each sample had run time of 18.5 minutes. A random standard solution was run after every 10 samples as a measure of quality control. Intrada AA $3 \mu \mathrm{m}$, $100 \times 3.0 \mathrm{~mm}$ column (Chrom Tech, Apple Valley, MN) was used, and an LCMS-8050, a triple quadrupole LC-MS/MS (Shimadzu Corporation, Kyoto, Japan) was applied in this work. Overall, optimization process of KYN and TRP measurements was followed as described previously. ${ }^{26,27}$ The $\mathrm{KT}$ ratio was obtained by dividing plasma concentration of $\mathrm{KYN}(\mu \mathrm{mol} / \mathrm{L})$ by the TRP concentration $(\mu \mathrm{mol} / \mathrm{L})$ and multiplying the quotient by $1,000 .{ }^{17,21}$ In this study, the $\mathrm{KT}$ ratio was used as a proxy of IDO activity.

Analyses of plasma and stool biomarkers by ELISA. Plasma samples were analyzed for C-reactive protein (CRP) (Immundiagnostik, Bensheim, Germany), alpha-1-acid glycoprotein (AGP) (Alpco, Salem, NH), and ferritin (ORGENTEC Diagnostika GmbH, 55129 Mainz, Germany) by using commercial ELISA kits. Zinc levels were determined by atomic absorption spectometry method. Stool samples were analyzed for neopterin (NEO) (GenWay, San Diego, CA), myeloperoxidase (Alpco), alpha-1-antitrypsin (AAT) (Biovendor, Chandler, NC), calprotectin (BÜHLMANN fCAL, Schönenbuch, Switzerland), and Reg1B (TechLab, Blacksburg, VA) using commercially available ELISAs according to kit manuals. The concentrations of each of the biomarkers were calculated against standards provided by the manufacturers.

Statistical analyses. Socioeconomic characteristics and demographic factors were described using frequencies with proportions when variables were categorical. Means and SDs were reported when quantitative variables were symmetrically distributed, whereas medians and interquartile ranges were used when quantitative variables were asymmetrically distributed. Differences in KYN, TRP, KT ratio, and other related variables were examined both pre- and post-intervention using Student's $t$-test when variables were continuous and parametric, whereas the Mann-Whitney or Wilcoxon tests were used for nonparametric and continuous variables.

Correlations among the KYN, TRP, and KT ratio with the biomarkers of EED, inflammation, and micronutrient status were examined using Spearman's correlation tests, both at baseline and endline. The correlation measures the strength and direction of association between different variables in our analysis. In this case, $P<0.05$ was considered as a measure of significance. Multivariable linear regression with generalized 
estimating equations (GEEs) was applied to test the association between $\mathrm{KT}$ ratio and $\mathrm{LAZ}$ while adjusting for age, gender, water/sanitation, assets, maternal education, and income (WAMI) (a combined variable to measure household socioeconomic status, access to improved water/sanitation, assets, maternal education, and income), maternal height, micronutrients, and fecal and inflammatory biomarkers. In the GEE model, unstructured correlation matrix was used along with Gaussian family, and the link function was the identity. Correlation matrix was applied under independence model criterion (QIC) value and chosen based on the lowest quasilikelihood. Variance inflation factor values were used to check the multicollinearity for all the models. Bivariate analysis was applied initially to identify the unadjusted effect of each explanatory variable on the outcome variable. Covariates were eventually selected for multivariable models if their association have significance of $<0.2$ with the outcome. Multivariable analyses were then applied using GEEs to test the associations considering adjustment for potential confounders. A probability of $<0.05$ was regarded statistically ignificant, and the strength of association was measured by the coefficient values and their 95\% Cls.

The GEE method allows specification of a working correlation matrix for the within-subject correlation of repeated responses collected from the same participants over time that eventually produced unbiased and more efficient regression parameters. $R$ version 3.5.1 was used for all the statistical analyses performed (https://www.r-project.org).

\section{RESULTS}

Sociodemographic and clinical characteristics. A total of 480 participants comprising 240 stunted and 240 at risk of stunting children were included in this study. The two groups differed significantly in age and gender. The mean $( \pm S D)$ age of stunted and at risk of stunting children were $14.8 \pm 2.12$ months and $14.3 \pm 2.13$ months, respectively (Table 1). Proportion of females was higher in children at risk of stunting (54.6\%) than in stunted children (44.6\%). The mean ( \pm SD) LAZ was $-2.79 \pm$ 0.63 for stunted children and $-1.56 \pm 0.29$ for at risk of stunting participants. Sociodemographic variables including length, weight, WAMI score, and maternal height were significantly lower in stunted than in at risk of being stunted children. The stunted children were also significantly more underweight (WAZ -2.15 versus -1.32 ) and wasted (WHZ -1.03 versus -0.80 ) than at risk of stunting participants. Besides, AAT level was found to be significantly different, whereas other stool and plasma biomarker levels did not differ significantly in the two groups.

Levels of plasma TRP, KYN, and $\mathrm{KT}$ ratio in study participants. Concentrations of TRP, KYN, and $\mathrm{KT}$ ratio as well as their comparisons in two groups are presented in Figure 1. Levels of TRP, KYN, and KT ratios were significantly higher in stunted participants than in at risk of being stunting participants, both at baseline and endline. In addition, the median concentration of $\mathrm{KYN}$ had significantly reduced to $3.88(0.26,7.56) \mu \mathrm{mol} / \mathrm{L}$ at endline from $4.58(3.64,5.45)$ $\mu \mathrm{mol} / \mathrm{L}$ at baseline, and the $\mathrm{KT}$ ratio was also significantly reduced to $92.8(6.66,247)$ from $104(80.9,131)$ for stunted participants. However, KT ratios were significantly higher in endline for at risk of stunting participants than for their counterparts. Significant differences were not found between baseline and endline TRP levels, both for stunted and at risk of stunting participants.

Correlation of TRP, KYN, and KT ratio with stool and plasma biomarkers. In this analysis, correlations between different indicators at baseline and endline were assessed using Spearman's rank correlation coefficient. Significant negative correlation was found between TRP and NEO both at baseline and endline (correlation coefficient, baseline $=-0.12$; endline $=-0.12$ ), whereas significant positive correlation was found for KYN at baseline (correlation coefficient $=0.17$ ). The $\mathrm{KT}$ ratio was positively correlated with NEO (coefficient,

TABLE 1

Baseline characteristics of the stunted and at risk of being stunted children at enrollment

\begin{tabular}{|c|c|c|c|}
\hline Indicators & Stunted $(n=240)$ & At risk of stunting $(n=240)$ & $P$-value \\
\hline \multicolumn{4}{|l|}{ Sociodemographic variables } \\
\hline Mean age (SD) (months) & $14.8(2.12)$ & $14.3(2.13)$ & 0.03 \\
\hline Female gender, $n(\%)$ & $107(44.6)$ & $131(54.6)$ & 0.028 \\
\hline Mean weight-for-age $Z$ score (SD) & $-2.15(0.772)$ & $-1.32(0.665)$ & $<0.001$ \\
\hline Mean length-for-age $Z$ score (SD) & $-2.79(0.628)$ & $-1.56(0.285)$ & $<0.001$ \\
\hline Mean weight-for-height $Z$ score (SD) & $-1.03(0.854)$ & $-0.806(0.862)$ & 0.005 \\
\hline Mean weight (SD) (kg) & $7.80(0.843)$ & $8.41(0.781)$ & $<0.001$ \\
\hline Mean length (SD) (cm) & $70.8(2.77)$ & $73.4(2.35)$ & $<0.001$ \\
\hline Mean WAMI score (SD) & $0.565(0.123)$ & $0.603(0.137)$ & 0.002 \\
\hline Mean maternal height (SD) (cm) & $148(5.45)$ & $150(4.83)$ & $<0.001$ \\
\hline \multicolumn{4}{|l|}{ Fecal biomarkers of EED } \\
\hline Median AAT [Q1, Q3] (mg/g) & $0.230(0.0600,0.530)$ & $0.340(0.125,0.565)$ & 0.005 \\
\hline Median calprotectin [Q1, Q3] $(\mu \mathrm{g} / \mathrm{g})$ & $544(307,1,010)$ & $529(283,979)$ & 0.902 \\
\hline Median MPO [Q1, Q3] (ng/mL) & $2,310(1,330,4,550)$ & $2,190(1,340,4,060)$ & 0.516 \\
\hline Median NEO [Q1, Q3] (nmol/L) & $2,330(1,110,3,430)$ & $2,190(1,220,3,660)$ & 0.572 \\
\hline Median Reg1B [Q1, Q3] ( $\mathrm{gg} / \mathrm{mL})$ & $62.7(33.3,87.5)$ & $66.4(33.1,92.5)$ & 0.152 \\
\hline \multicolumn{4}{|l|}{ Markers of systematic inflammation } \\
\hline Median AGP [Q1, Q3] (mg/dL) & $94.7(72.0,121)$ & $91.5(66.1,124)$ & 0.328 \\
\hline Median CRP [Q1, Q3] (mg/L) & $1.32(0.403,3.87)$ & $1.50(0.509,5.59)$ & 0.155 \\
\hline \multicolumn{4}{|l|}{ Micronutrients } \\
\hline Median ferritin [Q1, Q3] (ng/mL) & $10.5(5.24,21.1)$ & $12.1(6.53,22.9)$ & 0.139 \\
\hline Median zinc [Q1, Q3] (mg/L) & $0.790(0.720,0.875)$ & $0.790(0.710,0.860)$ & 0.845 \\
\hline
\end{tabular}



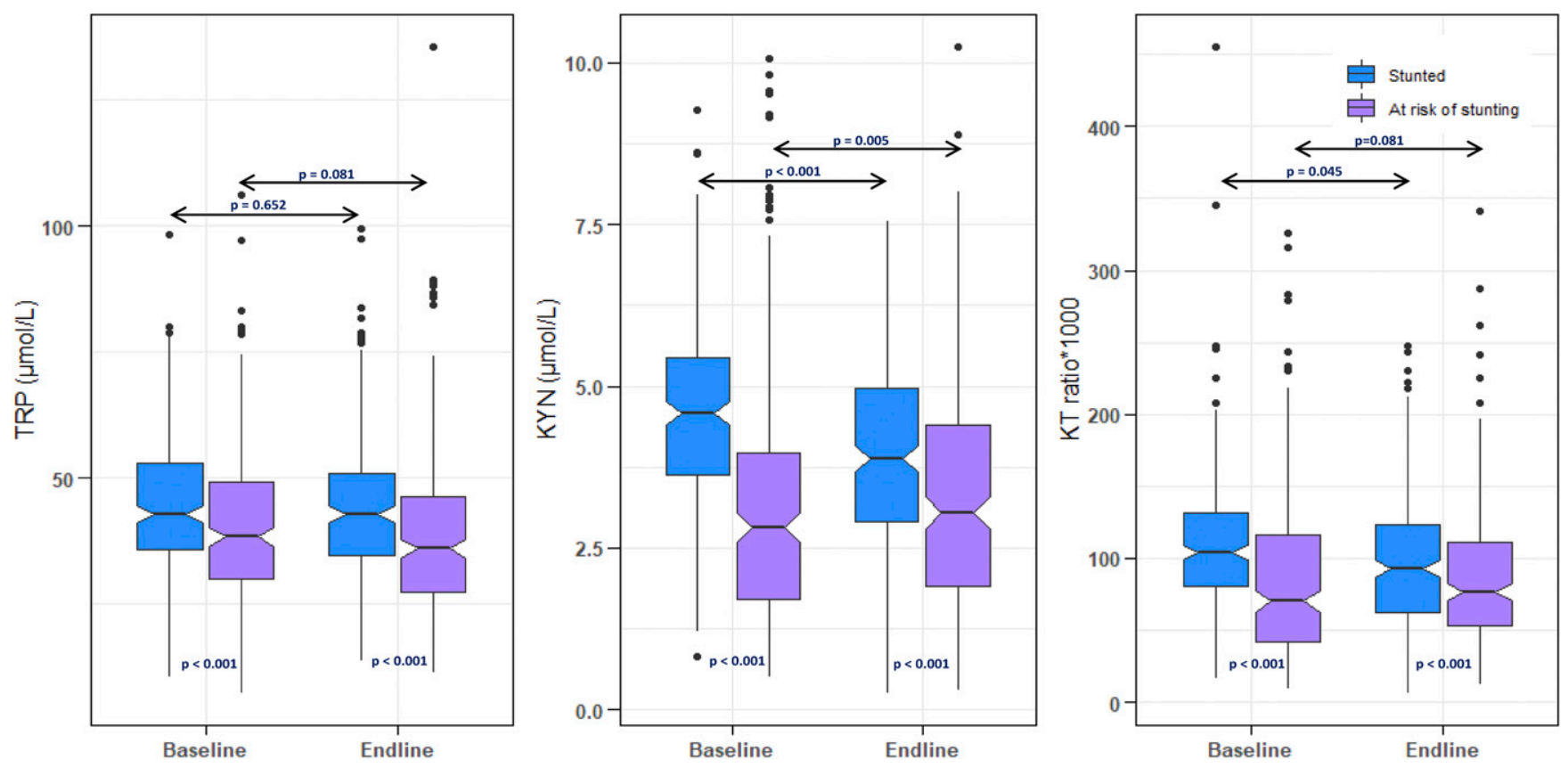

FIGURE 1. Baseline and endline concentrations of plasma tryptophan (TRP), kynurenine (KYN), and the KYN-TRP (KT) ratio in stunted and at risk of stunting children (or) the concentration of plasma TRP, KYN, and the KT ratio in stunted and at risk of stunting children, at baseline and endline. This figure appears in color at www.ajtmh.org.

baseline $=0.23$; endline $=0.10$ ). The $\mathrm{KT}$ ratio was negatively correlated with Reg1B at both time points, while being negatively correlated only at baseline with calprotectin and AAT. Among the plasma biomarkers, CRP was correlated negatively with TRP and positively with KT ratio, both at baseline and endline. Negative correlation was found for AGP and TRP at both time points (Supplemental Tables S1 and S2).

Association of KT ratio and other variables with LAZ. The analysis is longitudinal, with sample and anthropometric data collected from the same participants both in pre- and postintervention. All the children were considered including stunted and at risk of stunting to analyze the association of biomarkers with LAZ. Table 2 shows the association of KT ratio and other indicators with LAZ using GEEs. The KT ratio was found to be negatively associated with LAZ (coefficient = $-0.7 ; 95 \% \mathrm{Cl}=-1.13,-0.26 ; P$-value $=0.002$ ) on adjustment with the aforementioned confounders. Here, negative coefficient (-0.7) suggests that the mean decrease of LAZ by -0.7 unit for each one unit increase in the KT ratio. Significant positive association was observed in ferritin (coefficient = $1.62 ; 95 \% \mathrm{Cl}=0.32,2.92 ; P$-value $=0.015)$, maternal height (coefficient $=0.03 ; 95 \% \mathrm{Cl}=0.02,0.04 ; P$-value $=<0.001$ ), and the WAMI score (coefficient $=0.79 ; 95 \% \mathrm{Cl}=0.28,1.3 ; P$ value $=0.002$ ) with LAZ of the children. None of the EED and systemic inflammatory markers were found to be associated with LAZ of the children.

\section{DISCUSSION}

Linear growth failure in children is the most common manifestation of undernutrition worldwide. ${ }^{28}$ Impaired immune dysfunction resulting in recurrent infections may contribute to the high mortality rate associated with stunting, the causative pathways, which are still poorly understood. ${ }^{4}$ Moreover,

TABLE 2

Association of KT ratio and other variables with length-for-age Z-score score using generalized estimating equation at baseline and endline $(n=480)$

\begin{tabular}{|c|c|c|c|c|}
\hline Indicators & Unadjusted $\beta(95 \% \mathrm{Cl})$ & $P$-value & Adjusted $\beta(95 \% \mathrm{Cl})$ & $P$-value \\
\hline Age (months) & $0.03(0.02,0.03)$ & $<0.001$ & $0.02(0.02,0.03)$ & $<0.001$ \\
\hline Female gender & $0.17(0.03,0.31)$ & 0.015 & $0.14(0,0.27)$ & 0.044 \\
\hline $\mathrm{KT}$ ratio & $-0.8(-1.24,-0.36)$ & $<0.001$ & $-0.7(-1.13,-0.26)$ & 0.002 \\
\hline AAT $(\mathrm{mg} / \mathrm{g})$ & $0.02(-0.01,0.06)$ & 0.190 & $0.02(-0.01,0.06)$ & 0.172 \\
\hline Myeloperoxidase $(\mathrm{mg} / \mathrm{mL})$ & $-3.99(-8.12,0.14)$ & 0.059 & $-2.09(-6.08,1.9)$ & 0.305 \\
\hline Neopterin (mmol/L) & $0.16(-4.14,4.45)$ & 0.944 & - & - \\
\hline Calprotectin (g/g) & $0.76(-29.16,30.67)$ & 0.960 & - & - \\
\hline $\operatorname{Reg} 1 \mathrm{~B}(\mathrm{mg} / \mathrm{mL})$ & $-0.14(-0.47,0.19)$ & 0.403 & - & - \\
\hline AGP $(g / d L)$ & $-0.41(-0.95,0.13)$ & 0.135 & $-0.03(-0.57,0.51)$ & 0.900 \\
\hline $\operatorname{CRP}(g / L)$ & $1.27(-0.83,3.37)$ & 0.236 & - & - \\
\hline Ferritin $(\mu \mathrm{g} / \mathrm{mL})$ & $1.24(-0.08,2.56)$ & 0.066 & $1.62(0.32,2.92)$ & 0.015 \\
\hline Zinc (mg/L) & $-0.03(-0.22,0.16)$ & 0.744 & - & - \\
\hline Maternal height $(\mathrm{cm})$ & $0.03(0.02,0.05)$ & $<0.001$ & $0.03(0.02,0.04)$ & $<0.001$ \\
\hline WAMI score & $1.09(0.58,1.61)$ & $<0.001$ & $0.79(0.28,1.3)$ & 0.002 \\
\hline
\end{tabular}

KYN-TRP = kynurenine-tryptophan. 
unbalanced dietary intake and the mechanism by which it affects intestinal homeostasis are largely unrevealed. ${ }^{16}$ Identification of the mechanism and pathways associated with stunting are thus critical to distinguish the interrelated factors and to identify targets for intervention and mitigation. The TRP-IDO-KYN immunometabolic axis is a matter of great interest. In this study, we have quantified and analyzed the KYN pathway activation, also its association with linear growth of Bangladeshi children. Tryptophan metabolites have already been demonstrated to be promising indicators of TRP degradation induced by interferons. ${ }^{29}$ Our study results revealed that increased plasma $\mathrm{KT}$ ratio was found to be significantly and negatively associated with linear growth of the children in Bangladesh. Our findings also demonstrated that $\mathrm{KYN}$ and $\mathrm{KT}$ ratio were significantly elevated in stunted children compared with those among the at risk of being stunted children. In addition, KYN concentrations and KT ratios declined in stunted children receiving nutritional supplementation. However, we did not have a control group of stunted children to prove whether this supplementation led to the decrease in KYN and KT ratio. Hence, a control group (who did not receive supplementation) and comparison is essential to prove the supplementation effect. Our study also revealed that level of AAT was found to be significantly higher in nonstunted than in stunted children, whereas other stool and plasma biomarker levels did not differ between the groups. Alpha-1-anti-trypsin is an acute phase protein that indicates increased intestinal permeability and protein loss. ${ }^{30}$ Increased AAT in non-stunted children might explain the lower level of TRP found in this group. However, this finding complicates the overall notion of a physiologic pathway between intestinal permeability and chronic systemic inflammation.

Elevated KYN and KT ratio are markers of increased IDO activity. ${ }^{31}$ Increased KT ratio has shown to be associated with stunting earlier in other settings. ${ }^{22,32}$ Moreover, TRP and KYN/ TRP ratio associated with linear growth failure are accordant with prior studies that showed growth outcomes to be linked with several determinants including inadequate intake of nutrition, ${ }^{33,34} \mathrm{EED},{ }^{35,36}$ intestinal permeability, and disruption of the gut microbiota. ${ }^{37}$ Tryptophan metabolism mainly occurs via KYN pathway in the liver where the rate-limiting and first step is catalyzed by the hepatic TRP 2,3-dioxygenase (TDO) enzyme or the ubiquitous IDO. ${ }^{38}$ Microbial TRP uptake and metabolism may subvert this pathway. ${ }^{39}$ Whereas TDO is inherently active, IDO is activated by inflammatory cytokines. Therefore, in conditions with increased inflammation, TRP metabolism is switched from hepatic to extrahepatic tissues. ${ }^{38}$ Participants in the current study were slum dwellers and lived in a poor socioeconomic environment where frequent infection with pathogen is the norm. One of the adverse effects of elevated degradation and depletion of TRP in the body is the suppression of T-cell proliferation which is particularly evident in HIV infection. ${ }^{40}$ Other manifestations include weight loss, cognitive impairment, and mood disturbances. Cachexia or weight loss has also been found to be associated with low TRP levels in neoplasia. ${ }^{41}$ Increased catabolism of TRP through the KYN pathway may shift this EAA away from protein synthesis, and thus have contribution in muscle wasting and weight loss. ${ }^{41}$ Partitioning of AAs occur during immune system activation because of the competition between protein gain and utilization by the system. ${ }^{42}$ When supply of EAAs such as methionine, cysteine, threonine, and
TRP is limited, there is greater competition associated with the immune system for the AAs. ${ }^{42}$ The condition may also be associated with low socioeconomic conditions, intestinal infections, and multiple dietary factors that cause changes in microbiome. A murine model study found that $T$ cells cultured with IDO producing cells or low TRP media have lowered cell growth and increased cell apoptosis. ${ }^{17}$

Limitation of TRP in the diet could possibly have adverse effects on child growth through reduction of protein synthesis. $^{23}$ Nutritional deficiencies and other sociodemographic variables can also weaken the immune system, with potential effects on IDO activity. ${ }^{43}$ Children who are stunted often have insufficient TRP in their diets, and it has been exhibited that stunted children have lower levels of all nine EAAs in the serum, including TRP. ${ }^{14}$ Low serum TRP was found associated with biomarkers of gut barrier disruption and systemic inflammation and a predictor of growth failure in boys. ${ }^{44}$ Consequently, the syntheses of both proteins and lipids are restricted because of the mammalian target of rapamycin complex 1 (mTORC1) gene being repressed. $\mathrm{mTORC} 1$ is a master growth regulator, and the availability of AAs is exquisitely sensed via this pathway, for synthesis of proteins. ${ }^{10}$ Tryptophan activates mTORC1 and enhances the expression of tight junction proteins, involved in maintaining intestinal barrier function. ${ }^{6}$ Stunted children in lower income countries have scarce intake of EAAs, and this might hinder growth through the mTORC1 pathway.

In the current study, LAZ was found to be positively associated with plasma ferritin, maternal height, and WAMI score. Ferritin indicates the measure of iron stores in the body, if there is no concurrent infection. We adjusted the concentrations using AGP and CRP before analyses. As iron stores diminish, the ferritin value will decrease and cause immune disruption and stunting. Moreover, previous findings showed that intestinal ferritin loss may occur because of disruption of gut barrier function. ${ }^{30}$ Study also reported negative relationship between intestinal permeability and serum ferritin concentrations in young children and infants. ${ }^{45}$ The results of the WAMI score, maternal height, and stunting in this study are also consistent with previously published literature. ${ }^{46,47}$ None of the EED and systematic inflammatory biomarkers were associated with stunting. These results of EED and inflammatory biomarkers with linear growth are in line with other studies in same settings. ${ }^{48,49}$

We also assessed the correlation of TRP, KYN, and KT ratio with biomarkers of EED, systematic inflammatory biomarkers, and micronutrients. Our study revealed the positive correlation of NEO with KT ratio, both in baseline and endline, and KYN in baseline. Negative correlation was found for NEO with TRP at both time points. In some studies, concentrations of NEO were specifically quantified in different diseased conditions and showed a positive correlation with the KT ratio and KYN, and inversely with TRP. ${ }^{7,21,50,51}$ Neopterin is a marker of gut inflammation and immune system activation that is released by macrophages or monocytes, which activates the IDO pathway upon stimulation by interferon-gamma. ${ }^{52}$ So a rise in the NEO level gives a good estimate of the endogenous production of interferon-gamma and thus upregulation of the KYN pathway in EED and undernutrition. Possible mechanisms by which EED could influence TRP metabolism is through increased TRP breakdown by altered gut microbiota or through alterations in TRP transport by AA transporters. It is noted that dietary TRP is mostly absorbed by epithelial 
enterocytes using the neutral amino acid transporter/angiotensin-converting enzyme 2 transport pathway. ${ }^{16}$ One murine model study reported that deficiency in ACE2 results in increased risk of intestinal inflammation. Restoring TRP levels reduced inflammation and increased the production of antimicrobial peptides, indicating that ACE2 regulates dietary AA homeostasis, inflammation, gut microbial ecology, and innate immunity in a TRP-dependent manner. ${ }^{16}$ Our study result suggests that manipulation of TRP signaling may be pertinent to the pathophysiology of EED. ${ }^{22,53}$ A possible role of TRP signaling in regulating the villus structure and nutrient-sensing capability of immune cell has also been observed. ${ }^{54}$ A negative correlation was found for Reg1B with $\mathrm{KYN}$ and $\mathrm{KT}$ ratio both at baseline and endline, and again, negative correlation was found for AAT and calprotectin with $\mathrm{KYN}$ and KT ratio only at the endline. These negative correlations may be explained by the transient nature of EED markers in young children and need further elucidation with the KYN pathway. Moreover, KT ratio was positively correlated with AGP and CRP-biomarkers and measures of systemic inflammation. This finding is compatible with current thinking that intestinal infection, enterocyte function, and systemic immune response are closely related to EED. ${ }^{22}$ A recent study has shown that patients with flaky paint dermatosis had lower plasma TRP, and the authors had explained that the activity of IDO may be enhanced, because of increased CRP levels. ${ }^{55}$

\section{STRENGTHS AND LIMITATIONS}

There were some limitations in this study. First, IDO expression was not measured directly in the present study. However, studies have manifested that IDO activity is estimated best by the KT ratio. Second, increased expressions of interferon-gamma, interleukin-6, and interleukin-10 have been shown to be associated with increased catabolism of TRP by IDO. However, none of the mentioned cytokines were measured in our study. Besides this, the target population was children who were stunted or were at risk of stunting, and therefore by definition were not completely healthy. Finally, inability to include some of the important variables, such as birth weight, breastfeeding status, and morbidity were also major limitations of this study. The strength of this study includes a larger sample size. In addition, all the biomarkers were tested in a single batch to reduce potential batch effects and inter-tester variation.

In conclusion, the KT ratio was found to be significantly associated with growth in Bangladeshi children. In this study, as an estimation of increased IDO activity, the KT ratio came out as a possible measurement of childhood stunting. However, important questions remain to be unraveled that how TRP and KYN or KT ratio is reduced or elevated in the context of undernutrition and could be explored further in prospective studies. This alteration in the TRP pathway may reflect the effect of environmental stressors such as chronic inflammation, EED, low protein intake, or variations in the intestinal microbiota, because all of these can significantly affect the growth.

Received January 18, 2020. Accepted for publication September 26, 2020.
Published online November 23, 2020.

Note: Supplemental tables appear at www.ajtmh.org.

Acknowledgments: We thank the participants of the BEED study as well as the field and laboratory staff at icddr,b for their valuable contributions. icddr,b also gratefully acknowledges the following donors for their unrestrained support: Government of the People's Republic of Bangladesh, Global Affairs Canada (GAC), Canada; Swedish International Development Cooperation Agency (Sida) and the Department for International Development (UKAid). This research protocol is funded by the Bill \& Melinda Gates Foundation.

Financial support: This study was funded by the Bill \& Melinda Gates Foundation. The investment ID was OPP1136751. (https://www. gatesfoundation.org/How-We-Work/Quick-Links/GrantsDatabase/ Grants/2015/11/OPP1136751).

Disclosure: The funders had no role in the study design; collection, analysis, and interpretation of data; preparation, review, or approval of the manuscript; and decision to submit the manuscript.

Authors' addresses: Md. Amran Gazi, Subhasish Das, Md. Ashraful Alam, Shah Mohammad Fahim, Md. Mehedi Hasan, Farzana Hossaini, Mustafa Mahfuz, and Tahmeed Ahmed, Nutrition and Clinical Services Division, International Center for Diarrheal Disease Research, Bangladesh (icddr, b), Dhaka, Bangladesh, E-mails: amran.gazi@icddrb.org, subhasish.das@icddrb.org, mashraful@icddrb.org, mohammad. fahim@icddrb.org, md.hasan@icddrb.org, farzanasacky@gmail.com, mustafa@icddrb.org, and tahmeed@icddrb.org. Md. Abdullah Siddique, Md. Mamun Kabir, Zannatun Noor, and Rashidul Haque, Emerging Infection and Parasitology laboratory, International Centre for Diarrheal Disease Research, Bangladesh (icddr,b), Dhaka, Bangladesh, E-mails: abdullah@icddrb.org, mamunk@icddrb.org, zannatun@icddrb.org, and rhaque@icddrb.org.

This is an open-access article distributed under the terms of the Creative Commons Attribution (CC-BY) License, which permits unrestricted use, distribution, and reproduction in any medium, provided the original author and source are credited.

\section{REFERENCES}

1. UNICEF, 2018. Malnutrition Rates Remain Alarming: Stunting Is Declining Too Slowly while Wasting Still Impacts the Lives of Far Too Many Young Children. New York, NY: UNICEF.

2. De Onis M, Branca F, 2016. Childhood stunting: a global perspective. Matern Child Nutr 12: 12-26.

3. Mbuya MN, Humphrey JH, 2016. Preventing environmental enteric dysfunction through improved water, sanitation and hygiene: an opportunity for stunting reduction in developing countries. Matern Child Nutr 12: 106-120.

4. Bourke CD, Berkley JA, Prendergast AJ, 2016. Immune dysfunction as a cause and consequence of malnutrition. Trends Immunol 37: 386-398.

5. WHO, United Nations University, 2007. Protein and Amino Acid Requirements in Human Nutrition. Geneva, Switzerland: World Health Organization.

6. Wang H, Ji Y, Wu G, Sun K, Sun Y, Li W, Wang B, He B, Zhang Q, Dai Z, 2015. L-tryptophan activates mammalian target of rapamycin and enhances expression of tight junction proteins in intestinal porcine epithelial cells. J Nutr 145: 1156-1162.

7. Suzuki Y, Suda T, Furuhashi K, Suzuki M, Fujie M, Hahimoto D, Nakamura $\mathrm{Y}$, Inui N, Nakamura H, Chida K, 2010. Increased serum kynurenine/tryptophan ratio correlates with disease progression in lung cancer. Lung Cancer 67: 361-365.

8. Harper KM, Mutasa M, Prendergast AJ, Humphrey J, Manges AR, 2018. Environmental enteric dysfunction pathways and child stunting: a systematic review. PLoS Negl Trop Dis 12: e0006205.

9. Walson JL, Berkley JA, 2018. The impact of malnutrition on childhood infections. Curr Opin Infect Dis 31: 231-236.

10. Semba RD, Trehan I, Gonzalez-Freire M, Kraemer K, Moaddel R, Ordiz MI, Ferrucci L, Manary MJ, 2016. Perspective: the potential role of essential amino acids and the mechanistic target of rapamycin complex 1 (mTORC1) pathway in the pathogenesis of child stunting. Adv Nutr 7: 853-865. 
11. Pillai RR, Elango R, Ball RO, Kurpad AV, Pencharz PB, 2015. Lysine requirements of moderately undernourished school-aged Indian children are reduced by treatment for intestinal parasites as measured by the indicator amino acid oxidation technique. $J$ Nutr 145: 954-959.

12. Paton NI, Angus B, Chaowagul W, Simpson AJ, Suputtamongkol Y, Elia M, Calder G, Milne E, White NJ, Griffin GE, 2001. Protein and energy metabolism in chronic bacterial infection: studies in melioidosis. Clin Sci 100: 101-110.

13. Dror DK, Allen LH, 2011. The importance of milk and other animalsource foods for children in low-income countries. Food Nutr Bull 32: 227-243.

14. Semba RD, Shardell M, Ashour FAS, Moaddel R, Trehan I, Maleta KM, Ordiz MI, Kraemer K, Khadeer MA, Ferrucci L, 2016. Child stunting is associated with low circulating essential amino acids. EBioMedicine 6: 246-252.

15. Sanfilippo S, Imbesi R, Sanfilippo JS, 1995. Effects of a tryptophan deficient diet on the morphology of skeletal muscle fibers of the rat. Preliminary observations atneuroendocrinological and submicroscopical levels. Ital J Anat Embryol 100: 131-141.

16. Hashimoto $T$, Perlot $T$, Rehman A, Trichereau J, Ishiguro $\mathrm{H}$, Paolino M, SigI V, Hanada T, Hanada R, Lipinski S, 2012. ACE2 links amino acid malnutrition to microbial ecology and intestinal inflammation. Nature 487: 477-481.

17. Darcy CJ, Davis JS, Woodberry T, McNeil YR, Stephens DP, Yeo TW, Anstey NM, 2011. An observational cohort study of the kynurenine to tryptophan ratio in sepsis: association with impaired immune and microvascular function. PLoS One 6: e21185.

18. Rebnord EW, Strand E, Midttun Ø, Svingen GF, Christensen $\mathrm{MH}$, Ueland PM, Mellgren G, Njølstad PR, Tell GS, Nygård OK, 2017. The kynurenine: tryptophan ratio as a predictor of incident type 2 diabetes mellitus in individuals with coronary artery disease. Diabetologia 60: 1712-1721.

19. Favennec M, Hennart B, Caiazzo R, Leloire A, Yengo L, Verbanck M, Arredouani A, Marre M, Pigeyre M, Bessede A, 2015. The kynurenine pathway is activated in human obesity and shifted toward kynurenine monooxygenase activation. Obesity 23: 2066-2074.

20. Ciorba MA, 2013. Indoleamine 2, 3 dioxygenase (IDO) in intestinal disease. Curr Opin Gastroenterol 29: 146-152.

21. Huengsberg $M$, Winer JB, Gompels M, Round R, Ross J, Shahmanesh M, 1998. Serum kynurenine-to-tryptophan ratio increases with progressive disease in HIV-infected patients. Clin Chem 44: 858-862.

22. Kosek MN, Mduma E, Kosek PS, Lee GO, Svensen E, Pan WK, Olortegui MP, Bream JH, Patil C, Asayag CR, 2016. Plasma tryptophan and the kynurenine-tryptophan ratio are associated with the acquisition of statural growth deficits and oral vaccine underperformance in populations with environmental enteropathy. Am J Trop Med Hyg 95: 928-937.

23. Semba RD, Shardell M, Trehan I, Moaddel R, Maleta KM, Ordiz MI, Kraemer K, Khadeer M, Ferrucci L, Manary MJ, 2016. Metabolic alterations in children with environmental enteric dysfunction. Sci Rep 6: 28009.

24. Platts-Mills JA, Babji S, Bodhidatta L, Gratz J, Haque R, Havt A, McCormick BJ, McGrath M, Olortegui MP, Samie A, 2015. Pathogen-specific burdens of community diarrhoea in developing countries: a multisite birth cohort study (MAL-ED). Lancet Glob Health 3: e564-e575.

25. Mahfuz M, Das S, Mazumder RN, Rahman MM, Haque R, Bhuiyan MMR, Akhter H, Sarker MSA, Mondal D, Muaz SSA, 2017. Bangladesh Environmental Enteric Dysfunction (BEED) study: protocol for a community-based intervention study to validate non-invasive biomarkers of environmental enteric dysfunction. BMJ Open 7: e017768.

26. Möller M, Du Preez JL, Harvey BH, 2012. Development and validation of a single analytical method for the determination of tryptophan, and its kynurenine metabolites in rat plasma. $J$ Chromatogr B 898: 121-129.

27. Sun Z, Xing J, Khoo PY, Zhan Z, 2016. A Combined MRM and SIM Method for Direct Quantitative Determination of Amino Acids in Various Samples on LC/MS/MS. Sante Fe, NM: American Society for Mass Spectrometry.
28. Prendergast AJ, Humphrey $\mathrm{JH}, 2014$. The stunting syndrome in developing countries. Paediatr Int Child Health 34: 250-265.

29. Werner ER, Bitterlich G, Fuchs D, Hausen A, Reibnegger G, Szabo $\mathrm{G}$, Dierich MP, Wachter H, 1987. Human macrophages degrade tryptophan upon induction by interferon-gamma. Life Sci 41: 273-280.

30. Fahim SM, Das S, Sanin KI, Gazi MA, Mahfuz M, Islam MM, Ahmed T, 2018. Association of fecal markers of environmental enteric dysfunction with zinc and iron status among children at first two years of life in Bangladesh. Am J Trop Med Hyg 99: 489-494.

31. Pett SL, Kunisaki KM, Wentworth D, Griffin TJ, Kalomenidis I, Nahra R, Montejano Sanchez R, Hodgson SW, Ruxrungtham K, Dwyer D, 2017. Increased indoleamine-2, 3-dioxygenase activity is associated with poor clinical outcome in adults hospitalized with influenza in the INSIGHT FLU003Plus study. Open Forum Infect Dis 5: ofx228.

32. Moreau GB, Ramakrishnan G, Cook HL, Fox TE, Nayak U, Ma JZ, Colgate ER, Kirkpatrick BD, Haque R, Petri WA Jr., 2019. Childhood growth and neurocognition are associated with distinct sets of metabolites. EBioMedicine 44: 597-606.

33. Rah JH, Akhter N, Semba RD, De Pee S, Bloem MW, Campbell A, Moench-Pfanner R, Sun K, Badham J, Kraemer K, 2010. Low dietary diversity is a predictor of child stunting in rural Bangladesh. Eur J Clin Nutr 64: 1393-1398.

34. Ghosh S, Suri D, Uauy R, 2012. Assessment of protein adequacy in developing countries: quality matters. Br J Nutr 108: S77-S87.

35. Crane RJ, Jones KD, Berkley JA, 2015. Environmental enteric dysfunction: an overview. Food Nutr Bull 36: S76-S87.

36. Merchant A, Jones C, Kiure A, Kupka R, Fitzmaurice G, Herrera M, Fawzi W, 2003. Water and sanitation associated with improved child growth. Eur J Clin Nutr 57: 1562-1568.

37. Charbonneau MR, O'Donnell D, Blanton LV, Totten SM, Davis JC, Barratt MJ, Cheng J, Guruge J, Talcott M, Bain JR, 2016. Sialylated milk oligosaccharides promote microbiota-dependent growth in models of infant undernutrition. Cell 164: 859-871.

38. Badawy AA, 2017. Kynurenine pathway of tryptophan metabolism: regulatory and functional aspects. Int $J$ Tryptophan Res 10: 1178646917691938.

39. Ahmed T, Auble D, Berkley JA, Black R, Ahern PP, Hossain M, Hsieh A, Ireen S, Arabi M, Gordon JI, 2014. An evolving perspective about the origins of childhood undernutrition and nutritional interventions that includes the gut microbiome. Ann New York Acad Sci 1332: 22-38.

40. Vujkovic-Cvijin I, Dunham RM, Iwai S, Maher MC, Albright RG, Broadhurst MJ, Hernandez RD, Lederman MM, Huang Y, Somsouk M, 2013. Dysbiosis of the gut microbiota is associated with HIV disease progression and tryptophan catabolism. Sci Transl Med 5: 193ra91.

41. Chen Y, Guillemin GJ, 2009. Kynurenine pathway metabolites in humans: disease and healthy states. Int $J$ Tryptophan Res 2: $1-19$.

42. Kampman-van de HoekE, Jansman AJ, van den Borne JJ, van der Peet-Schwering CM, van Beers-Schreurs H, Gerrits WJ, 2015. Dietary amino acid deficiency reduces the utilization of amino acids for growth in growing pigs after a period of poor health. $J$ Nutr 146: 51-58.

43. Badawy AA, 2017. Tryptophan availability for kynurenine pathway metabolism across the life span: control mechanisms and focus on aging, exercise, diet and nutritional supplements. Neuropharmacology 112: 248-263.

44. Guerrant RL, Leite AM, Pinkerton R, Medeiros PH, Cavalcante PA DeBoer M, Kosek M, Duggan C, Gewirtz A, Kagan JC, 2016. Biomarkers of environmental enteropathy, inflammation, stunting, and impaired growth in children in northeast Brazil. PLOS One 11: e0158772.

45. Berant M, Khourie M, Menzies IS, 1992. Effect of iron deficiency on small intestinal permeability in infants and young children. $J$ Pediatr Gastroenterol Nutr 14: 17-20.

46. Psaki SR, Seidman JC, Miller M, Gottlieb M, Bhutta ZA, Ahmed T, Ahmed AS, Bessong P, John SM, Kang G, 2014. Measuring socioeconomic status in multicountry studies: results from the eight-country MAL-ED study. Popul Health Metr 12: 8. 
47. MAL-ED Network Investigators, 2017. Childhood stunting in relation to the pre-and postnatal environment during the first 2 years of life: the MAL-ED longitudinal birth cohort study. PLOS Med 14: e1002408.

48. Campbell RK, Schulze K, Shaikh S, Mehra S, Ali H, Wu L, Raqib R, Baker S, Labrique A, West KP Jr., 2017. Biomarkers of environmental enteric dysfunction among children in rural Bangladesh. J Pediatr Gastroenterol Nutr 65: 40-46.

49. Hossain M, Nahar B, Haque M, Mondal D, Mahfuz M, Naila NN, Gazi M, Hasan M, Haque NMS, Haque R, 2019. Serum adipokines, growth factors, and cytokines are independently associated with stunting in Bangladeshi children. Nutrients 11:1827.

50. Dusek T, Orhalmi J, Sotona O, Krcmova LK, Javorska L, Dolejs J, Paral J, 2018. Neopterin, kynurenine and tryptophan as new biomarkers for early detection of rectal anastomotic leakage. Videosur Other Miniinvasive Tech 13: 44-52.

51. Murr C, Gerlach D, Widner B, Dierich MP, Fuchs D, 2001. Neopterin production and tryptophan degradation in humans infected by Streptococcus pyogenes. Med Microbiol Immunol 189: 161-163.
52. Zuo H, Ueland PM, Ulvik A, Eussen SJ, Vollset SE, Nygård O, Midttun $\varnothing$, Theofylaktopoulou D, Meyer K, Tell GS, 2016. Plasma biomarkers of inflammation, the kynurenine pathway, and risks of all-cause, cancer, and cardiovascular disease mortality: the Hordaland health study. Am J Epidemiol 183: 249-258.

53. Metz R, Rust S, DuHadaway JB, Mautino MR, Munn DH, Vahanian NN, Link CJ, Prendergast GC, 2012. IDO inhibits a tryptophan sufficiency signal that stimulates mTOR: a novel IDO effector pathway targeted by D-1-methyl-tryptophan. Oncoimmunology 1: 1460-1468.

54. Louis-Auguste J, Besa E, Zyambo K, Munkombwe D, Banda R, Banda T, Watson A, Mayneris-Perxachs J, Swann J, Kelly P, 2019. Tryptophan, glutamine, leucine, and micronutrient supplementation improves environmental enteropathy in Zambian adults: a randomized controlled trial. Am J Clin Nutr 110: 1240-1252.

55. Maltos AL, Portari GV, Moraes GV, Monteiro MCR, Vannucchi H, da Cunha DF, 2015. Niacin metabolism and indoleamine 2, 3-dioxygenase activation in malnourished patients with flaky paint dermatosis. Nutrition 31: 890-892. 